\title{
Direct Analysis in Real Time Mass Spectrometry of Potential By-Products from Homemade Nitrate Ester Explosive Synthesis
}

\author{
Edward Sisco ${ }^{a *}$, Thomas P. Forbes ${ }^{a}$ \\ ${ }^{a}$ National Institute of Standards and Technology, Materials Measurement Science Division, \\ Gaithersburg, Maryland 20899, United States
}

Author to whom correspondence should be sent:

Edward Sisco

National Institute of Standards and Technology

100 Bureau Drive

Mail Stop 6371

Gaithersburg, MD 20899

United States

E-mail: edward.sisco@nist.gov

Telephone: +1-301-975-2093

Fax: +1-301-417-1321 
Keywords: Explosives, Nitrate Esters, Direct Analysis in Real Time, Mass Spectrometry, Forensic Science

\begin{abstract}
This work demonstrates the coupling of direct analysis in real time (DART) ionization with timeof-flight mass spectrometry (MS) in an off-axis configuration for the trace detection and analysis of potential partially nitrated and dimerized by-products of homemade nitrate ester explosive synthesis. Five compounds relating to the synthesis of nitroglycerin (NG) and pentaerythritol tetranitrate (PETN) were examined. Deprotonated ions and adducts with molecular oxygen, nitrite, and nitrate were observed in the mass spectral responses of these compounds. A global optimum temperature of $350^{\circ} \mathrm{C}$ for the by-products investigated here, enabled single nanogram to sub nanogram trace detection. Matrix effects were examined through a series of mixtures containing one or more compounds (sugar alcohol precursors, by-products, and/or explosives) across a range of mass loadings. The explosives MS responses experienced competitive ionization in the presence of all by-products. The magnitude of this influence corresponded to both the degree of by-product nitration and the relative mass loading of the by-product to the explosive. This work provides a characterization of potential by-products from homemade nitrate ester synthesis, including matrix effects and potential challenges that might arise from the trace detection of homemade explosives (HMEs) containing impurities. Detection and understanding of HME impurities and complex mixtures may provide valuable information for the screening and sourcing of homemade nitrate ester explosives.
\end{abstract}




\section{Introduction}

Rapid explosives detection with minimal sample preparation remains an area of interest to forensic science, homeland security, and military agencies. The class and state of explosives investigated varies widely across these fields and may include military-grade secondary explosives, homemade explosives (HMEs) such as nitrate esters and peroxides, primary fuel-oxidizer explosives, improvised explosive device (IED) components, synthesis precursors, and detonated decomposition products. There is an extensive literature base involving the detection of military-grade nitrated organic explosives, e.g. RDX or TNT, including common binders and plasticizers [1]. As nonstate terrorism has evolved, there is an increased need to explore the detection of HMEs, including their precursors, synthesis by-products, impurities, and decomposition products. The detection of these compounds and their mixtures may enhance chemical libraries or provide valuable information into the route of synthesis and source materials. Because of the potential additional information provided, it is crucial to not only detect these compounds but also understand how they behave in mixtures with the explosives.

One of the major classes of HMEs, nitrate ester explosives, represents a broad category of organic explosives, both military-grade and homemade, including compounds such as nitroglycerin (NG), pentaerythritol tetranitrate (PETN), and erythritol tetranitrate (ETN). Nitrate esters are synthesized through nitration of a sugar alcohol (polyol) precursor, as simplified in Figure 1. This synthesis reaction may result in several potential by-products, namely partially nitrated and polymeric nitrated species. While a number of nitrate ester explosives exist, this work focuses on the nitration of glycerol (leading to NG) and pentaerythritol (leading to PETN) and their potential synthesis impurities. Both explosives have been synthesized militarily and illicitly, and are studied extensively [1-3]. A number of studies, mainly using liquid chromatography mass spectrometry (LC/MS), have identified impurities from NG and PETN synthesis, including partially nitrated and dimer species [4-7]. Partially nitrated species, such as 1,3dinitroglycerin (DNG), can occur from incomplete nitration of the sugar alcohol due to lack of available free nitrate ions, abrupt cessation of the reaction, or other improper synthesis conditions $[2,8]$. Partially nitrated species can also form via hydrolysis of a fully nitrated nitrate ester [2]. Likewise, improper termination of the reaction process can cause the formation of dimer, trimer, and other polymeric species. Dimer species can also be formed from impurities in the starting sugar alcohol, such as the presence of diglycerol $[9,10]$. In addition to by-products formed during the reaction, a number of 
decomposition products, also partially nitrated species, may be created $[4,6,7,9]$. The relative amount of these impurities and degradation products are dependent on the route of synthesis and/or length of decomposition. Relative levels of DNG in groundwater samples containing NG were $50 \%$ to $100 \%$ that of NG [4]. Similarly, the presence of PETriN in degradation samples ranged from $20 \%$ to $100 \%$ that of PETN, while neat samples contained only approximately $0.1 \%$ [7].

This work highlights the characterization of potential by-products and decomposition products of NG and PETN using direct analysis in real time (DART) mass spectrometry (MS). DART-MS is an increasingly used ambient ionization (AI)-MS technique in forensic science laboratories [11], requiring minimal or no sample preparation, and has previously demonstrated detection of nitrate ester explosives [12-14] and their sugar alcohol precursors [15]. Extensive details of DART ionization pathways and analysis can be found in the literature $[11,13,16]$. The characterization presented here focused on identifying the mass spectral response of potential by-products and decomposition products. The compounds investigated included: 1-mononitroglycerin (1-MNG), 2mononitroglycerin (2-MNG), 1,3-dinitroglycerin (DNG), pentaerythritol trinitrate (PETriN), and dipentaerythritol hexanitrate (diPEHN). In addition to the DART-MS characterization of these compounds, analysis of mixtures with their corresponding explosive (NG or PETN) and/or precursors (glycerol or pentaerythritol) was conducted. All compounds were readily detected in negative ionization mode as adducts with anions such as molecular oxygen, nitrite, and nitrate detectable in the low nanogram to sub nanogram range. The detection of explosives from mixtures with by-products demonstrated increasing ionization competition as by-product nitration increased. The large volatility difference between monomers and dimers produced significantly less competitive ionization with the explosive signal. Competitive ionization for the mixtures examined increased as the mass ratio of by-product to explosive increased.

\section{Materials and Methods}

Liquid chromatography-mass spectrometry (LC-MS) Chromasolv ${ }^{\circledR}$ grade acetonitrile was purchased from Sigma-Aldrich (St. Louis, MO, USA) and used for sample dilution. ${ }^{\S}$ 1-mononitroglycerin (1-MNG), 2-mononitroglycerin (2-MNG), and 1,3-dinitroglycerin (DNG) were purchased from Cerilliant (Round Rock, TX, USA) while pentaerythritol trinitrate (PETriN), and dipentaerythritol hexanitrate (diPEHN) were purchased from AccuStandard (New Haven, CT, USA) at a concentration of $1 \mathrm{mg} / \mathrm{mL}$ or $0.1 \mathrm{mg} / \mathrm{mL}$, in 
either methanol or acetonitrile, and further diluted in acetonitrile to desired concentrations. Nitric acid (Sigma-Aldrich) diluted in acetonitrile was used as an additional precursor in mixture analysis. Glycerol (gly) and pentaerythritol (PE) were purchased in pure form from Sigma Aldrich and dissolved/diluted in acetonitrile. Nitroglycerin (NG) and pentaerythritol tetranitrate (PETN) were purchased as $1 \mathrm{mg} / \mathrm{mL}$ standards from AccuStandard (New Haven, CT, USA) and diluted in acetonitrile. Samples were deposited by directly pipetting $1 \mu \mathrm{L}-2 \mu \mathrm{L}$ aliquots of the desired compounds onto polytetrafluoroethylene (PTFE, Teflon) wells, coated on glass slides (Tekdon Inc., Mayakka City, FL, USA). Polyethylene glycol 600 (PEG 600) (SigmaAldrich) diluted in acetonitrile was used as the MS tuning compound.

For this work a Vapur ${ }^{\circledR}$ interface (IonSense, Saugus, MA, USA) was used, coupling the DART ionization source (lonSense) sampling region and the time-of-flight mass spectrometer (JEOL AccuTOF JMS-T100LP, JEOL USA, Peabody, MA, USA). The Vapur ${ }^{\circledR}$ allowed for off-axis sampling [15] (45 ${ }^{\circ}$ with respect to the sample surface) by pulling the aerosolized/vaporized sample towards the mass spectrometer inlet at a rate of approximately $3.8 \mathrm{~L} / \mathrm{min}$. Off-axis analysis, completed by pulling the gas flow into the mass spectrometer instead of allowing direct transmission of the DART gas into the mass spectrometer permitted larger samples and sample areas to be analyzed. Off-axis analysis resulted in a decrease in atmospheric noise, potentially increasing sensitivity. In the off-axis system used, the substrate was mounted vertically on a manual 3-axis stage and positioned less than $1 \mathrm{~mm}$ away from the ceramic tip on the $\operatorname{Vapur} \AA$ inlet. The DART source was then turned to $45^{\circ}$, relative to the MS inlet, for analysis. Base mass spectrometer parameters included a $350{ }^{\circ} \mathrm{C}$ DART gas stream temperature, $100{ }^{\circ} \mathrm{C}$ orifice temperature, $\pm 20 \mathrm{~V}$ orifice 1 voltage, $\pm 5 \mathrm{~V}$ ring and orifice 2 voltages, $\pm 2,000 \mathrm{~V}$ detector voltage, $\pm 400 \mathrm{~V}$ peaks voltage and a mass scan range of $40 \mathrm{~m} / \mathrm{z}$ to $800 \mathrm{~m} / \mathrm{z}$. Unless otherwise noted, $100 \mathrm{ng}$ aliquots of the compounds were deposited onto PTFE coated glass slides and interrogated for $45 \mathrm{~s}$ to $60 \mathrm{~s}$ at $0.5 \mathrm{~s} / \mathrm{scan}$ for analyses.

\section{Results and Discussion}

\subsection{Mass Spectral Characteristics and Sensitivities}

Five compounds of interest (1-MNG, 2-MNG, DNG, PETriN, and diPEHN) were analyzed directly off PTFE coated glass slides, using DART-MS, to obtain characteristic mass spectra in both negative and positive ionization modes. Peaks of interest were identified and mass assignments were made based upon accurate mass measurements 
( $\pm 0.005 \mathrm{Da}$ mass accuracy), which are highlighted in Table 1. No identifiable peaks attributed to the compounds of interest were detected at the $100 \mathrm{ng}$ level in positive ionization mode; therefore, the remainder of this discussion focuses on negative ionization mode. The response of these compounds was dependent upon the first orifice voltage, which controls declustering and the extent of fragmentation/adduct formation.

At relatively low orifice voltage $(-10 \mathrm{~V})$, these compounds readily formed adducts with a number of ionic species generated through DART ionization pathways [17], including molecular oxygen $\left[\mathrm{M}+\mathrm{O}_{2}\right]^{-}$, nitrite $\left[\mathrm{M}+\mathrm{NO}_{2}\right]^{-}$, and nitrate $\left[\mathrm{M}+\mathrm{NO}_{3}\right]^{-}$. Dimers of the compounds with these adducts $\left(\left[2 \mathrm{M}+\mathrm{O}_{2}\right]^{-},\left[2 \mathrm{M}+\mathrm{NO}_{2}\right]^{-}\right.$, and $\left.\left[2 \mathrm{M}+\mathrm{NO}_{3}\right]^{-}\right)$were also observed (Figures 2 and 3 and Table 1). In all instances, "M" denotes an intact molecule of the by-product. Numerous studies have demonstrated the adduct formation of nitrated explosives, e.g., nitrate esters and nitroamines, with available anions using DART and other ambient ionization techniques [13,18,19]. The extent of sugar alcohol nitration determined the dominant ionization pathway between deprotonation or nitrate adduct formation. Sugar alcohols (no nitration) have demonstrated preferential ionization through deprotonation $[14,15]$. Similarly, the singularly nitrated species, 1-MNG and 2MNG, with multiple hydroxyl groups demonstrated observable deprotonated molecular, $[\mathrm{M}-\mathrm{H}]^{-}$, and dimer ions, $[2 \mathrm{M}-\mathrm{H}]^{-}$, (Figure 2 and Table 1). As the extent of nitration increased, e.g., DNG, nitrate adduct formation became the dominant pathway (Figure 2). The larger and more stable PETriN molecule also readily formed dimer adduct species, as shown in Figure 2. In addition to these common DART-formed adducts and dimer species, all of the compounds also adducted with a previously identified $\mathrm{HCO}_{4}{ }^{-}$ion $(\mathrm{HO}-$ $\mathrm{CO}_{3}{ }^{-}$), observed with DART ionization [15].

An elevated orifice 1 voltage $(-30 \mathrm{~V})$ was utilized to investigate reduced nonpreferential adduct and dimer formation through increased frequency of collisions between gas molecules and ions in the differentially pumped region (Figure 2B, 2D, 3B, and 3D). Tuning this potential difference beneficially simplified the mass spectra to a single, or several, channels of higher intensity, which enhanced detection of these compounds. DNG, PETriN, and diPEHN exhibited simplified mass spectra that further increased the signal of the base peak $\left(\left[\mathrm{M}+\mathrm{NO}_{3}\right]^{-}\right)$while decreasing the intensity of the remaining peaks (Figure 2 and 3). 1-MNG and 2-MNG readily fragmented into predominantly nitrate ions, indicating greater thermal instability than the more nitrated species. 
Low-level detection of these compounds by DART-MS was examined by measuring the signal-to-noise ratio of the base peak $\left(\left[\mathrm{M}+\mathrm{NO}_{3}\right]^{-}\right)$at decreasing mass loadings. In all instances, replicate measurements were made to ensure the observed signal-to-noise ratio was reproducible both inter-day and intra-day. However, the range of physicochemical properties of these compounds, and most specifically volatility, first necessitated an optimization of the DART gas stream temperature.

DART gas stream temperature drives desorption of sample off the substrate surface. Optimization between efficient desorption, signal duration (versus response of the mass spectrometer), and thermal degradation/decomposition was investigated. Figure 4 demonstrates the DART-MS response of the by-products investigated here and previous work on the sugar alcohol precursors/nitrate ester explosives, for gas stream temperatures of $150{ }^{\circ} \mathrm{C}$ to $450{ }^{\circ} \mathrm{C}$. The maximum MS response was dependent upon the size and volatility of the compound, with the more volatile MNG species readily detected at $150{ }^{\circ} \mathrm{C}$ and the less volatile diPEHN species undetectable until temperatures were greater than $250{ }^{\circ} \mathrm{C}$. A gas stream temperature of $350{ }^{\circ} \mathrm{C}$ demonstrated global optimal (or near optimal) signal across the majority of compounds and was previously found to be the optimal temperature for the detection of the corresponding sugar alcohols [15]; therefore, $350{ }^{\circ} \mathrm{C}$ was used for the remainder of this study. Signal consistency, as measured by \%RSD (relative standard deviation), largely increased with increasing temperature, as desorption of the compounds likely became more efficient. For example, PETriN decreased in \%RSD from $71 \%$ at $150{ }^{\circ} \mathrm{C}$ to $10 \%$ at $400{ }^{\circ} \mathrm{C}$. Variation in signal typically fell within $10 \%$ to $30 \%$ RSD and was due to a number of factors including differences in sample alignment and variations from the ionization source.

Once an optimal gas stream temperature was established, low-level sensitivity measurements were made by analyzing decreasing mass loadings of each by-product. For the by-products examined, signal-to-noise ratios between $3: 1$ and 6:1 were measured in the hundreds of picograms to single nanogram regime at a first orifice voltage of $-20 \mathrm{~V}$ and DART gas stream temperature of $350{ }^{\circ} \mathrm{C}$. The relatively low volatility of diPEHN enabled further improvements to sensitivity at elevated DART gas stream temperature above $350{ }^{\circ} \mathrm{C}$ if necessary.

\subsection{Analysis of Mixtures - Precursors, By-Products and Explosives}

The detection of nitrate ester explosives in the presence of synthesis precursors and by-products was also investigated. Competitive ionization phenomena of simultaneously 
interrogated species have been demonstrated for a range of ambient ionization and atmospheric pressure ionization platforms [14,15,20,21]. For this investigation, explosives, by-products, and their sugar alcohol precursors were analyzed both individually and as mixtures of equal mass. Figure 4 displays the peak area of the nitrate adduct for by-products and explosives, as well as the deprotonated molecular ion peak for sugar alcohols. Results for the NG series demonstrated marginal decrease in explosive signal in a complex mixture of by-products and precursors (statistically insignificant, Student's T-test, $95 \%$ confidence level) (Figure 5A). The compound likely responsible for the observed competitive ionization was DNG, as evidenced in the binary DNG-NG mixture (Figure 5A). The observed competition was preliminarily attributed to the relative affinity for nitrate adduct formation between DNG and NG. Additionally, the single hydroxyl functional group of DNG may reduce steric hindrance for nitrate adduct formation relative to NG. Ion suppression may result through the desorption or ionization processes. Gas phase competitive ionization of binary mixtures using plasma-based ionization platforms has been studied with a plug injection system [22]. A similar investigation will be the focus of future work. Consistent with results obtained from other sugar alcohol explosive mixtures [14], the presence of glycerol revealed a minimal but statistically significant (Student's T-Test, $95 \%$ confidence level) effect on the signal of NG. The discrimination of these compound classes was attributed to differences in volatility and nitrate ion affinities between the two [15].

The PETN series (Figure 5B) exhibited similar results. Again, the by-product with a single remaining hydroxyl group (PETriN) incrementally decreased the signal for the explosive compound. This decrease in PETN signal was statistically insignificant. Both PETriN and DNG displayed an increased nitrate adduct response in the presence of the explosive, credited to additional availability of nitrate ions from the thermal decomposition of the explosive during the desorption process. The dimer species, diPEHN, displayed little effect on the explosive signal. However, the presence of PETN significantly suppressed the diPEHN response (statistically different, Student's T-test, $99.9 \%$ confidence level). Future experiments will measure the relative affinities of each compound combination to nitrate adduct formation. In both instances demonstrated here, the signal of the explosive was suppressed by less than $20 \%$ when present in complex multi-component mixtures containing by-products and precursors. These mixtures represent extremes for potential HME synthesis formulations. A more realistic synthesis might yield by-product and precursor impurities at much lower concentrations than the 
explosive, unlike the equal mass mixtures investigated here. The competitive ionization dependence on relative mixture component abundances was investigated next.

A series of mixtures were deposited, at trace levels, with increasing amounts of byproduct relative to explosive, from explosive with no by-product to explosive with byproduct present at a 10-fold greater mass loading (Figure 6). In all instances, the mass of the explosive remained constant. Three to five replicates of each loading were completed, monitoring the signals for the nitrate adducts of both the explosive and byproduct. NG (Figure 6A) exhibited a significant drop in signal with increasing levels of both MNG and DNG. As expected from Figure 4, DNG produced greater NG suppression than MNG due to its higher nitrate affinity. The NG response fell below background levels in a binary mixture with DNG at 10x higher mass loading. While the likelihood of a ten-fold excess of DNG in a trace explosive residue is very low, the competitive ionization demonstrated highlights the crucial nature of understanding how these compounds interact with one another when sampled.

The effect of PETN by-products on the explosive signal was far less pronounced than that observed with NG (Figure 6B). The presence of diPEHN produced less than a $20 \%$ decrease in PETN signal, while the presence of PETriN produced an approximately 50 $\%$ loss in signal for equal mass loadings. Given the significant differences in volatility and nitrate affinity between diPEHN and PETriN, the differences in competitive ionization with PETN were expected. In addition, this study was conducted as a function of component mass and the larger diPEHN yields fewer molecules per mass than PETriN and PETN, compounded the differences.

\section{Conclusions}

Partially nitrated and dimer by-product species, formed during the synthesis of nitrate ester explosives were readily detected by DART-MS. All five compounds examined produced strong and specific negative ion mass spectra at low $(-10 \mathrm{~V}$ to $-30 \mathrm{~V})$ first orifice plate voltages. DART-MS gas stream temperature profiles highlighted increasing optimal temperature with increasing by-product size. Larger molecules produced a stronger signal at higher temperatures due to increased thermal stability and decreased desorption. A global optimum temperature of $350{ }^{\circ} \mathrm{C}$, led to trace detection in the hundreds of picograms to single nanogram range. Additional studies explored the matrix effects of these compounds on the detection of their corresponding explosives. Mixtures of the by-products with the explosive compounds highlighted increased signal 
suppression with increasing nitration. DNG and PETriN demonstrated the strongest matrix effects, e.g., competitive ionization, on their corresponding nitrate ester explosive. The dimer species, diPEHN, minimally affected the PETN signal, potentially due to lower nitrate affinity and decreased volatility. DART-MS experiments demonstrated elevated matrix effects and signal suppression of the explosives for increasing by-product mass. Future work will focus on identifying methods to further increase sensitivity of this technique and, more importantly, understanding the role competitive and preferential ionization effects play in detection of nitrate ester explosives from realistic homemade explosive synthesis residues. 


\section{Acknowledgements}

The U.S. Department of Homeland Security Science and Technology Directorate sponsored a portion of the production of this material under Interagency Agreement IAA HSHQDC-12-X00024 with the National Institute of Standards and Technology

(NIST).

§Certain commercial products are identified in order to adequately specify the procedure; this does not imply endorsement or recommendation by National Institute of Standards and Technology, nor does it imply that such products are necessarily the best available for the purpose. 


\section{References}

[1] D. Moore, Instrumentation for trace detection of high explosives, Rev Sci Instrum. 75 (2004) 2499-2512.

[2] R. Boschan, R.T. Merrow, R.W. van Dolah, The Chemistry of Nitrate Esters, Chem. Rev. 55 (1955) 485-510. doi:10.1021/cr50003a001.

[3] J.P. Agrawal, R. Hodgson, Organic Chemistry of Explosives, John Wiley \& Sons, 2007.

[4] R. Martel, A. Bellavance-Godin, R. Lévesque, S. Côté, Determination of Nitroglycerin and Its Degradation Products by Solid-Phase Extraction and LC-UV, Chromatographia. 71 (2009) 285-289. doi:10.1365/s10337-009-1415-2.

[5] X. Zhao, J. Yinon, Identification of nitrate ester explosives by liquid chromatographyelectrospray ionization and atmospheric pressure chemical ionization mass spectrometry, J. Chromatogr. A. 977 (2002) 59-68. doi:10.1016/S0021-9673(02)01349-3.

[6] D.M. Baaske, N.N. Karnatz, J.E. Carter, High-performance liquid chromatographic assay for partially nitrated glycerins in nitroglycerin, J. Pharm. Sci. 72 (1983) 194-196. doi:10.1002/jps.2600720226.

[7] H. Brust, A. van Asten, M. Koeberg, A. van der Heijden, C.-J. Kuijpers, P. Schoenmakers, Pentaerythritol tetranitrate (PETN) profiling in post-explosion residues to constitute evidence of crime-scene presence, Forensic Sci. Int. 230 (2013) 37-45. doi:10.1016/j.forsciint.2013.03.042.

[8] S.K. Yasuda, Identification and determination of impurities in pentaerythritol tetranitrate, J. Chromatogr. A. 51 (1970) 253-260. doi:10.1016/S0021-9673(01)96861-X.

[9] Stability Studies on Pentaerythritol Tetranitrate - Om Reddy - 2004 - Propellants, Explosives, Pyrotechnics - Wiley Online Library, (n.d.). http://onlinelibrary. wiley.com/doi/10.1002/prep.19920170610/abstract;jsessionid=23D512C 145BA4440532D79EAB55D1699.f04t01 (accessed September 29, 2015).

[10] W.J. A, Preparation and purification of nitrated pentaerythritols, US2294592 A, 1942. http://www.google.com/patents/US2294592 (accessed September 29, 2015).

[11] J.A. Laramée, R.B. Cody, J.M. Nilles, H.D. Durst, Forensic Application of DARTTM (Direct Analysis in Real Time) Mass Spectrometry, in: R.D.B.R. former S. Chemist (Ed.), Forensic Anal. Cut. Edge New Methods Trace Evid. Anal., John Wiley \& Sons, Inc., 2007: pp. 175195. http://onlinelibrary.wiley.com/doi/10.1002/9780470166932.ch7/summary (accessed April 26, 2012).

[12] J.M. Nilles, T.R. Connell, S.T. Stokes, H. Dupont Durst, Explosives Detection Using Direct Analysis in Real Time (DART) Mass Spectrometry, Propellants Explos. Pyrotech. 35 (2010) 446-451. doi:10.1002/prep.200900084.

[13] E. Sisco, J. Dake, C. Bridge, Screening for trace explosives by AccuTOF ${ }^{\mathrm{TM}}$-DART®: an indepth validation study, Forensic Sci. Int. 232 (2013) 160-168. doi:10.1016/j.forsciint.2013.07.006.

[14] T.P. Forbes, E. Sisco, Trace detection and competitive ionization of erythritol tetranitrate in mixtures using direct analysis in real time mass spectrometry, Anal. Methods. 7 (2015) 3632-3636. doi:10.1039/C4AY02694B.

[15] E. Sisco, T.P. Forbes, Rapid detection of sugar alcohol precursors and corresponding nitrate ester explosives using direct analysis in real time mass spectrometry, The Analyst. 140 (2015) 2785-2796. doi:10.1039/c4an02347a.

[16] E.S. Chernetsova, G.E. Morlock, I.A. Revelsky, DART mass spectrometry and its applications in chemical analysis, Russ. Chem. Rev. 80 (2011) 235-255. doi:10.1070/RC2011v080n03ABEH004194.

[17] J.H. Gross, Direct analysis in real time--a critical review on DART-MS, Anal. Bioanal. Chem. 406 (2014) 63-80. doi:10.1007/s00216-013-7316-0. 
[18] M. Morelato, A. Beavis, P. Kirkbride, C. Roux, Forensic applications of desorption electrospray ionisation mass spectrometry (DESI-MS), Forensic Sci. Int. 226 (2013) 10-21. doi:10.1016/j.forsciint.2013.01.011.

[19] Z. Takáts, J.M. Wiseman, R.G. Cooks, Ambient mass spectrometry using desorption electrospray ionization (DESI): instrumentation, mechanisms and applications in forensics, chemistry, and biology, J. Mass Spectrom. 40 (2005) 1261-1275. doi:10.1002/jms.922.

[20] R. Knochenmuss, F. Dubois, M.J. Dale, R. Zenobi, The Matrix Suppression Effect and Ionization Mechanisms in Matrix-assisted Laser Desorption/Ionization, Rapid Commun. Mass Spectrom. 10 (1996) 871-877. doi:10.1002/(SICl)10970231(19960610)10:8<871::AID-RCM559>3.0.CO;2-R.

[21] K. Tang, J.S. Page, R.D. Smith, Charge competition and the linear dynamic range of detection in electrospray ionization mass spectrometry, J. Am. Soc. Mass Spectrom. 15 (2004) 1416-1423. doi:10.1016/j.jasms.2004.04.034.

[22] J.T. Shelley, G.M. Hieftje, lonization matrix effects in plasma-based ambient mass spectrometry sources, J. Anal. At. Spectrom. 25 (2010) 345. doi:10.1039/b923564g. 


\section{Figure Captions}

Figure 1. Reaction pathway and potential by-products that occur during the synthesis and decomposition of nitrate ester explosives. In this example, pentaerythritol, pentaerythritol tetranitrate, and related compounds are shown.

Figure 2. Representative negative ion mass spectra of 2-mononitroglycerin (2-MNG) (A. \& B.) and 1,3-dinitroglycerin (DNG) (C. \& D.) in negative ionization mode at $-10 \mathrm{~V}$ (top) and $-30 \mathrm{~V}$ (bottom) orifice 1 voltage. Select peaks of interest are highlighted.

Figure 3. Representative negative ion mass spectra of pentaerythritol trinitrate (PETriN) (A. \& B.) and dipentaerythritol hexanitrate (C. \& D.) in negative ionization mode at -10 V (top) and -30 $\mathrm{V}$ (bottom) orifice 1 voltage. Select peaks of interest are highlighted.

Figure 4. MS response of the examined compounds (nitrate adduct signal) to increasing DART gas stream temperature. Data points and uncertainty represent the average and standard deviation of four to five replicate measurements. DART-MS responses of the explosives (PETN and NG) and precursor sugar alcohols (PE and Gly) are adapted with permission from ref 15. Copyright 2014 Royal Society of Chemistry.

Figure 5. Effect of precursors and by-products on the explosive signal of NG (A.) and PETN (B.). The bars represent the integrated peak area of the by-product and explosive nitrate adducts or the precursor sugar alcohol deprotonated molecular ions. Error bars represent the standard deviation of 3 to 5 replicate measurements. "Pure" represents the signal obtained for each compound interrogated individually. "Mixture" represents the signal obtained for all four compounds interrogated simultaneously. The remaining three categories are combinations of the explosive and a single second component interrogated simultaneously.

Figure 6. Effect of the presence of increasing amount of by-products on the signal of NG (A.) and PETN (B.). Mass ratio is the mass of by-product to the mass of explosive, which remained constant throughout the experiment. Error bars represent the standard deviation of 3 to 5 measurements. 


\section{Table Captions}

Table 1. Structure, accurate masses, and mass spectral assignments for the nitrate ester by-products examined. In all cases, the accurate masses and mass spectral assignments were calculated using MassCenter software. 


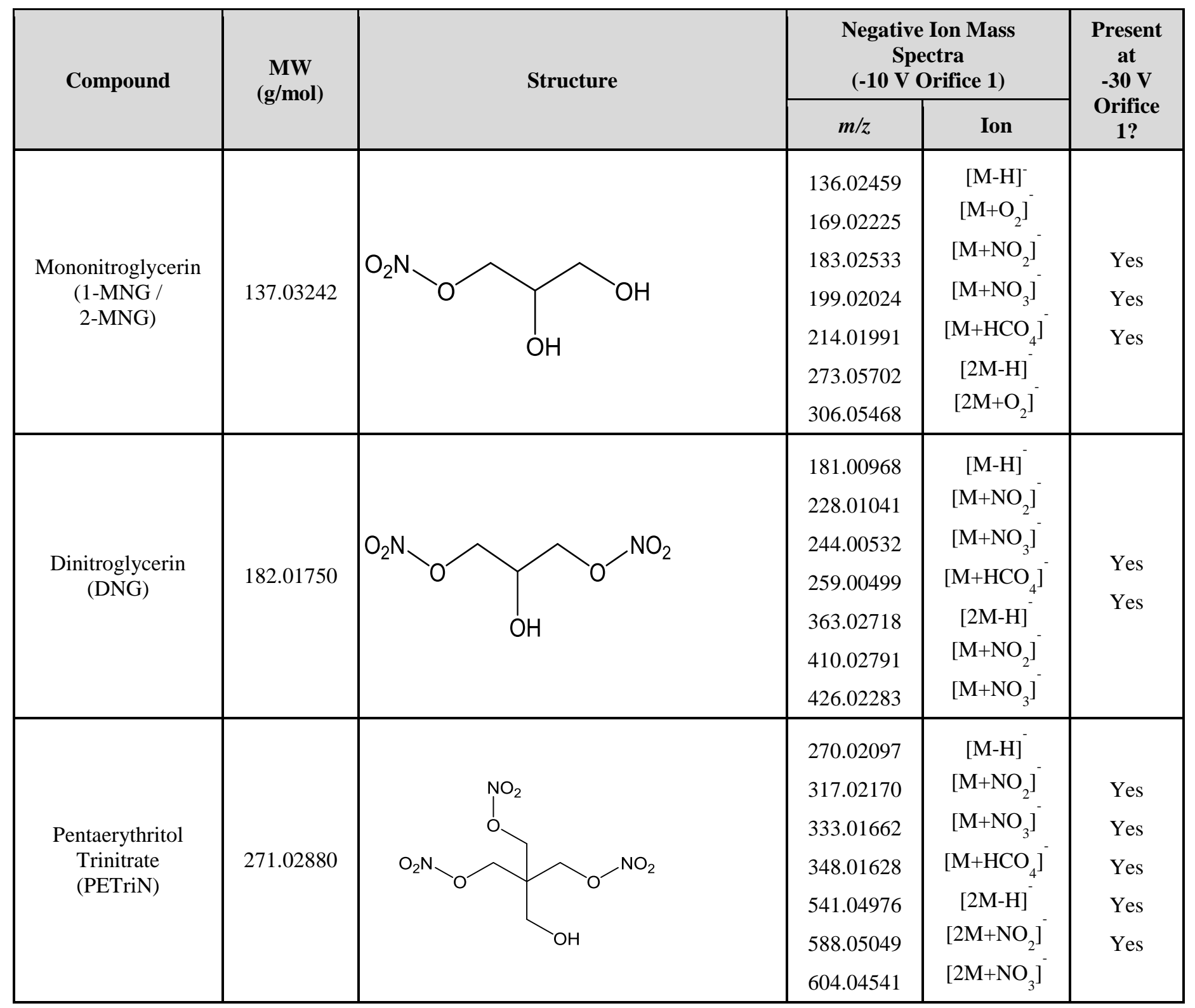




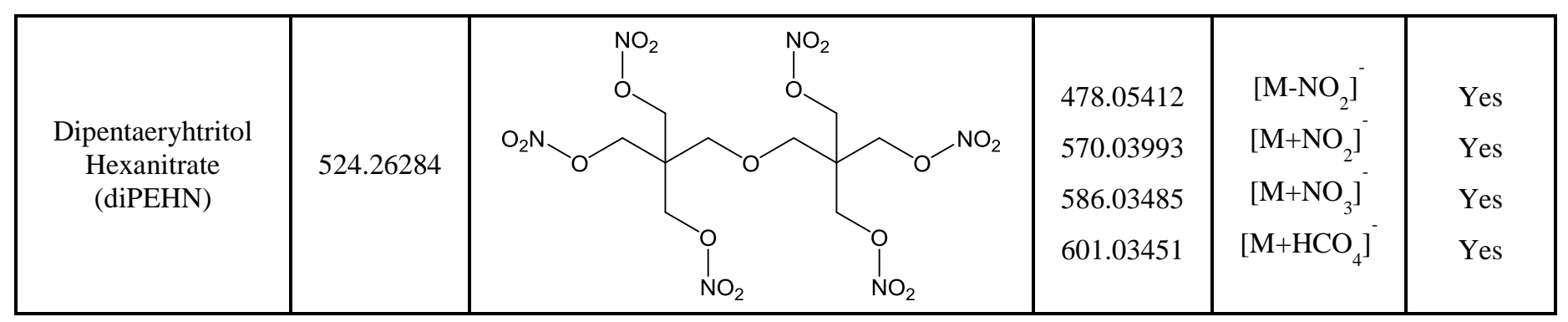




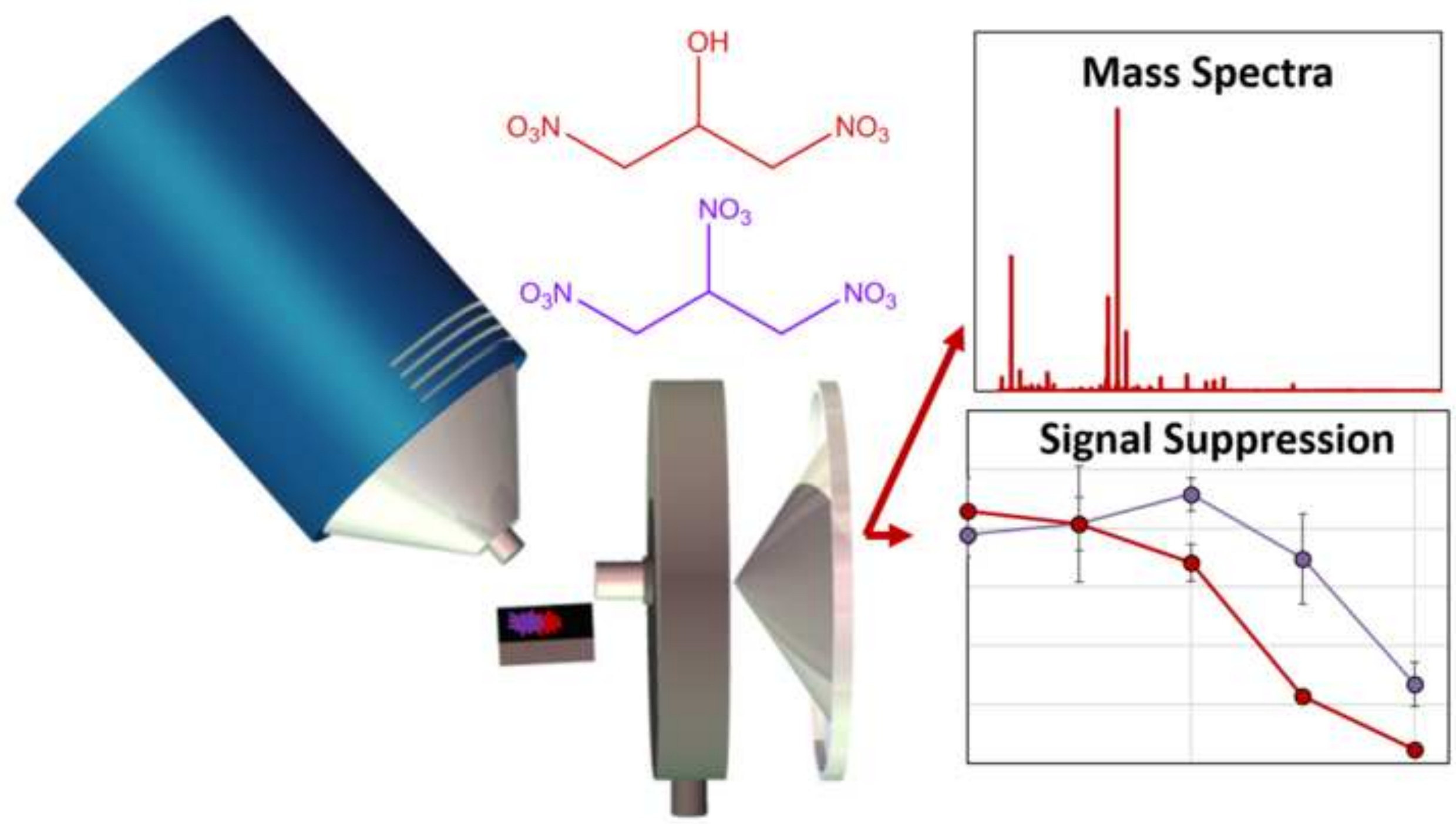




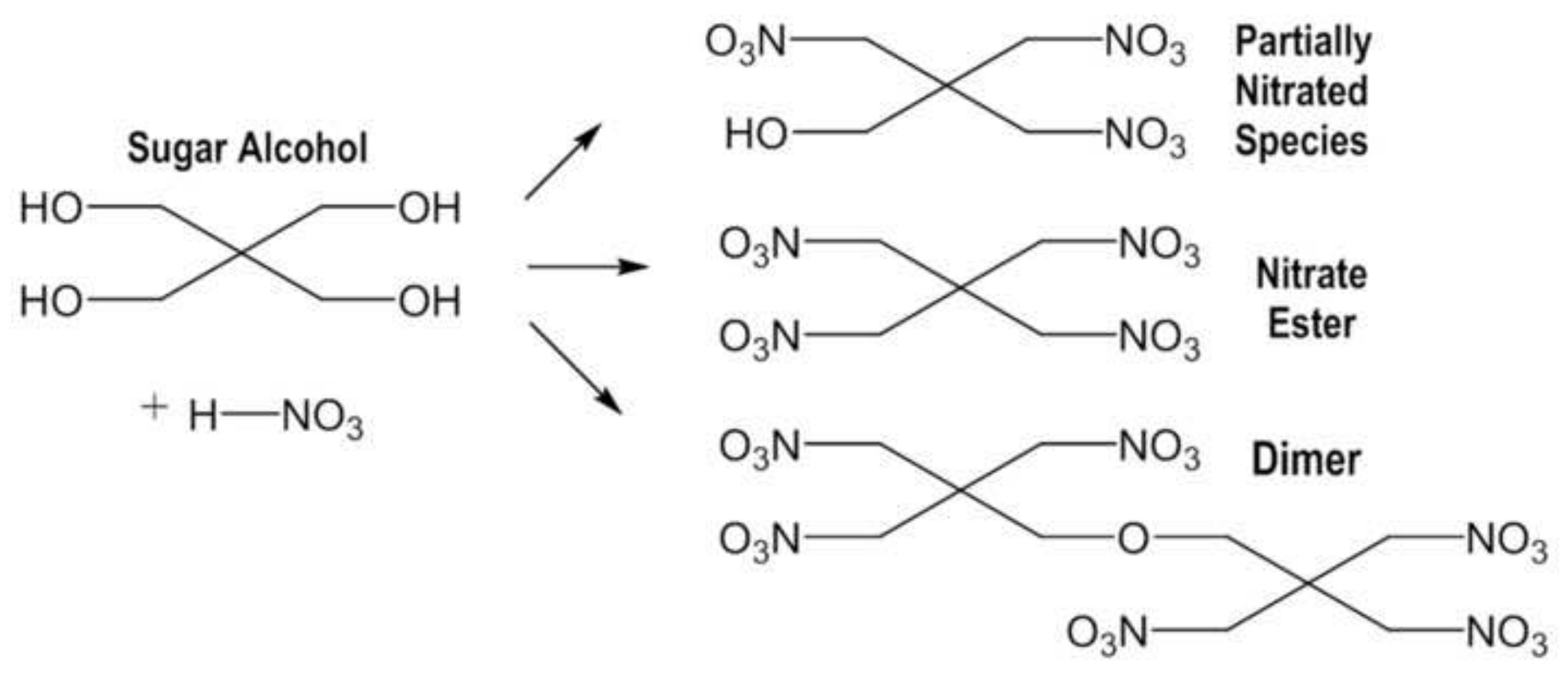




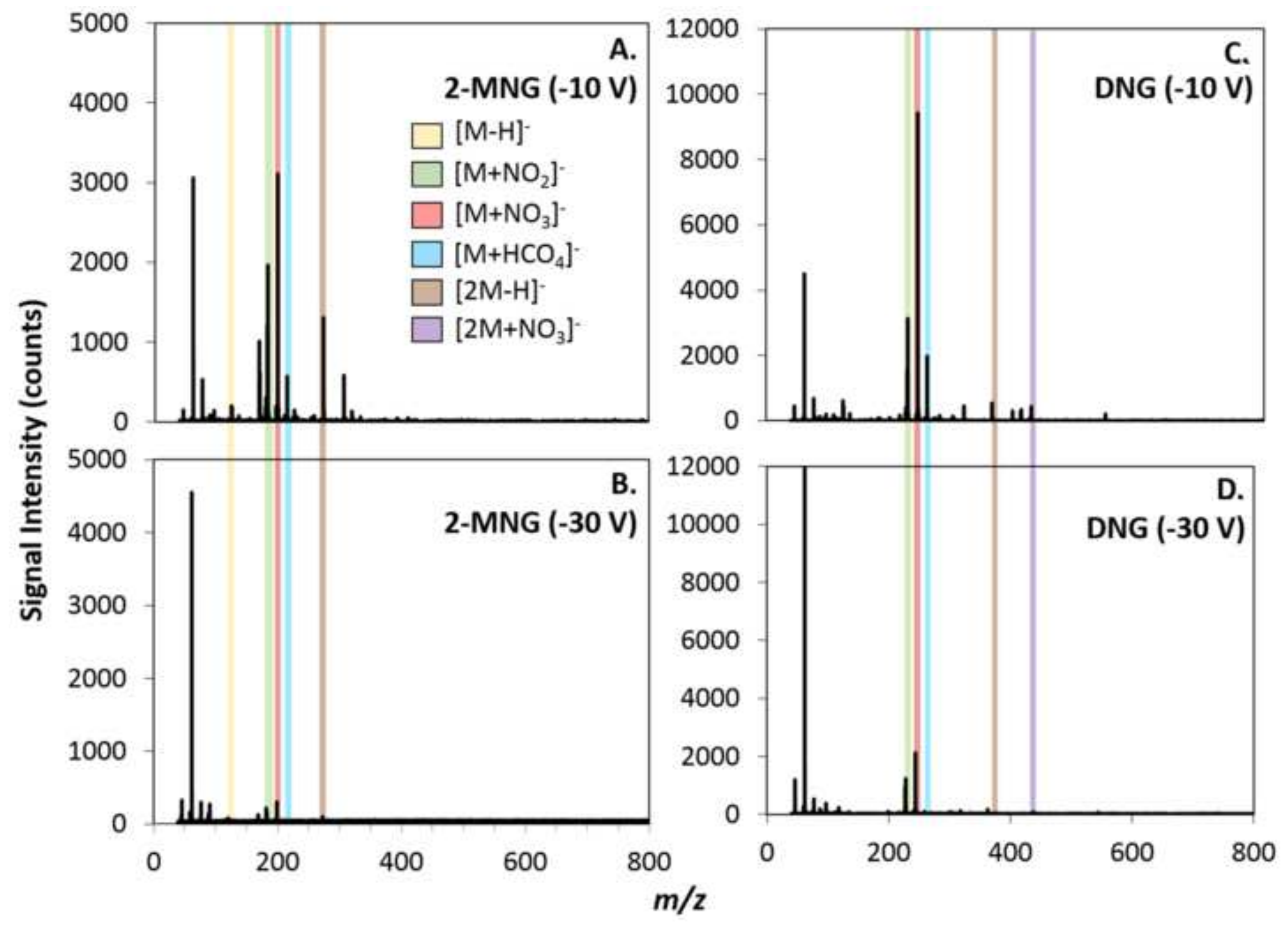




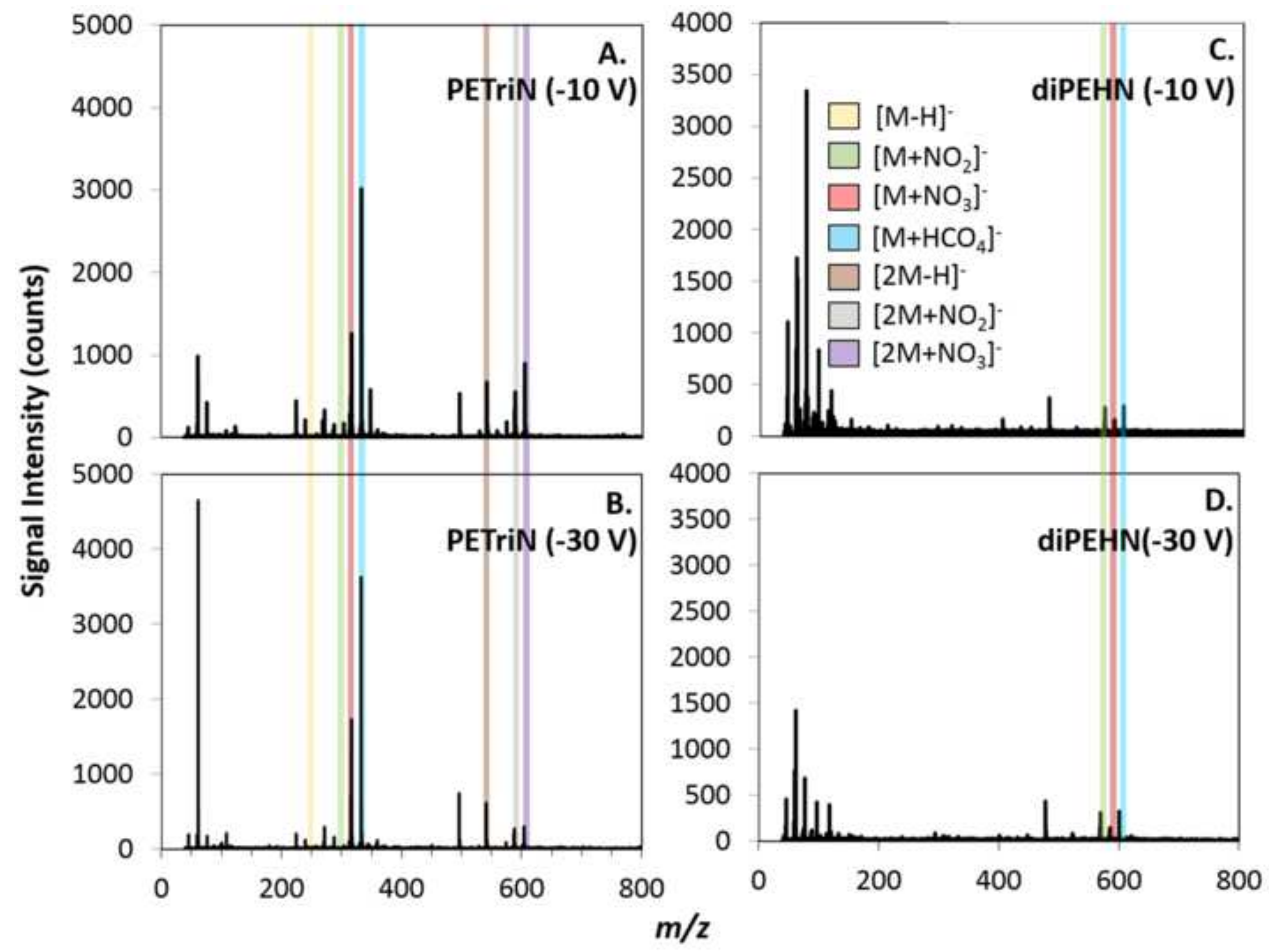




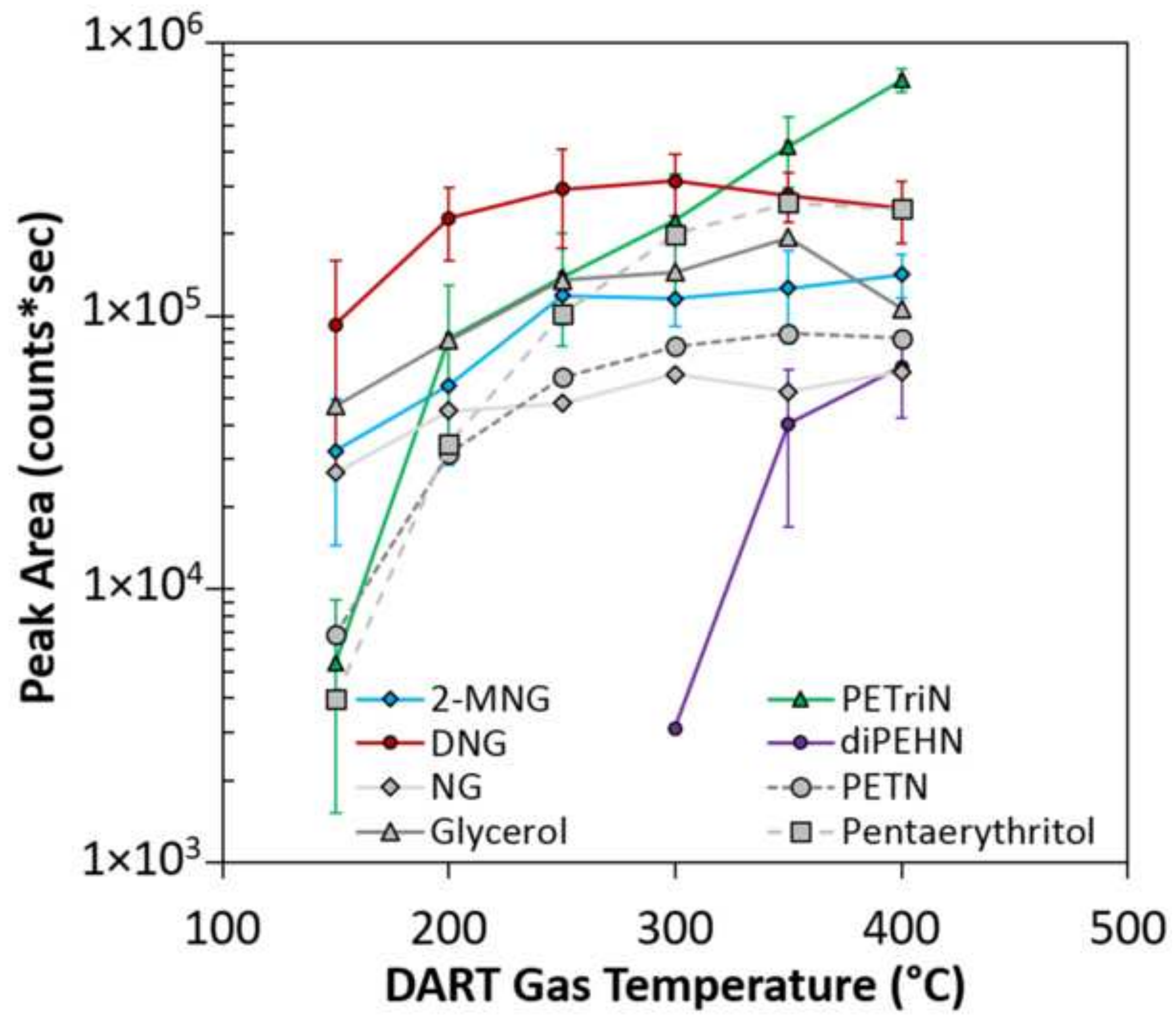



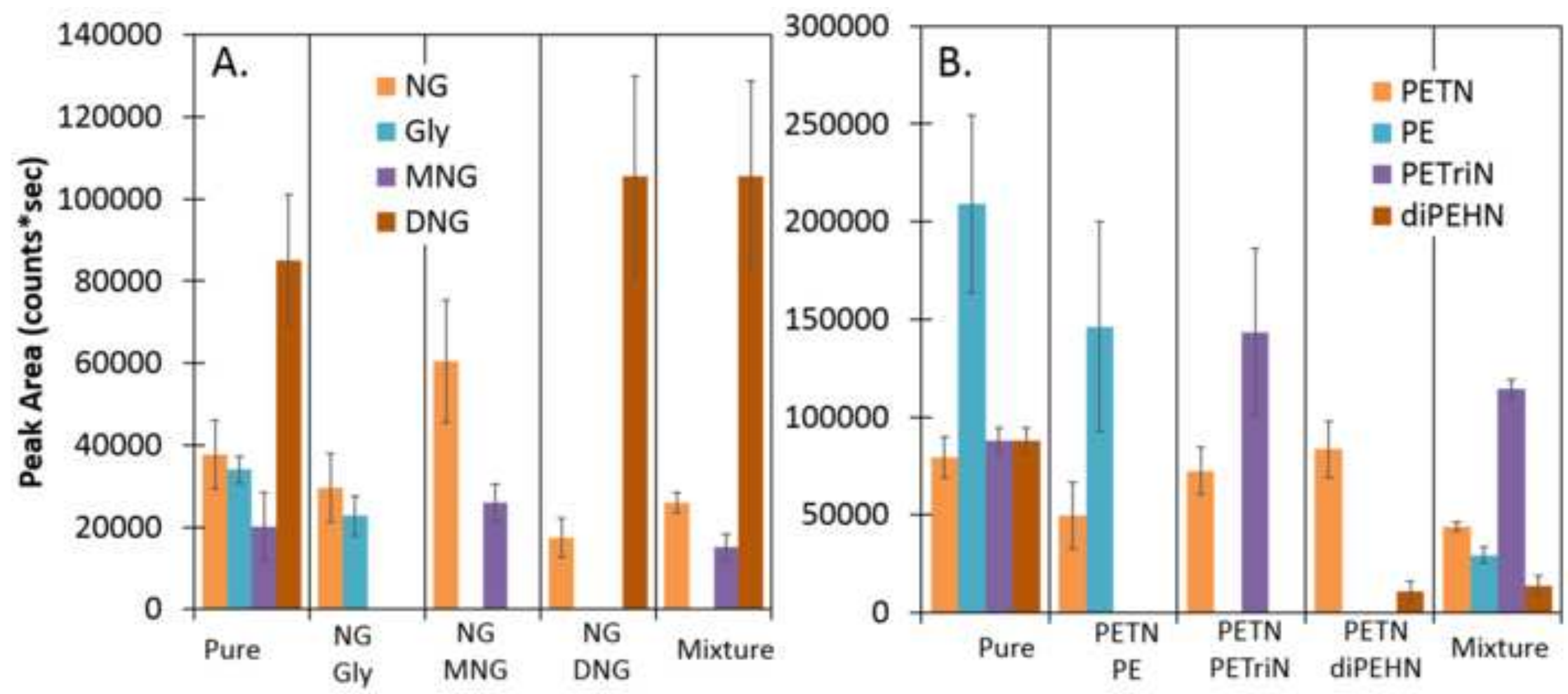


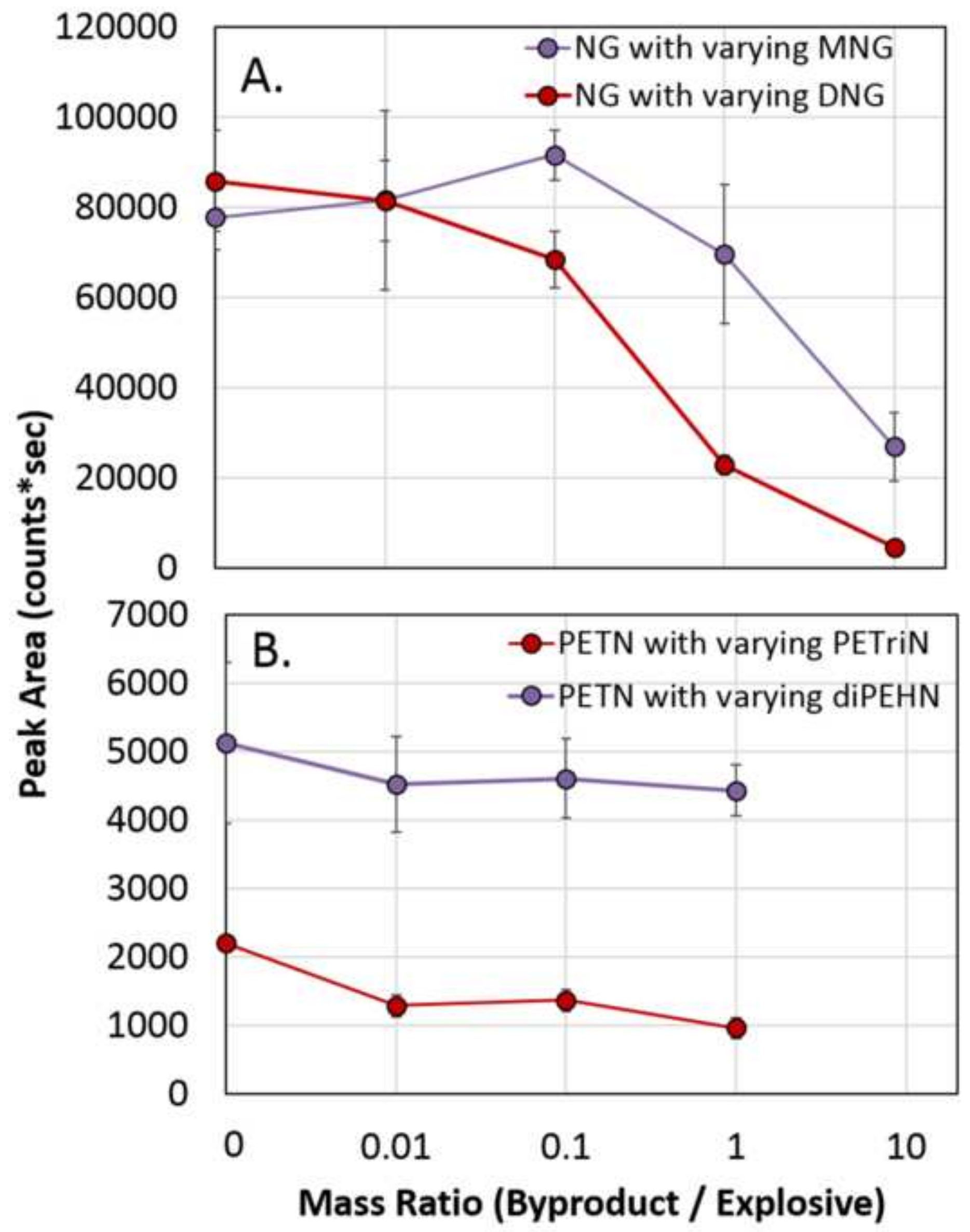

